\title{
PREDIÇÃO DE VALORES GENÉTICOS POR MEIO DE MODELOS ROBUSTOS
}

\author{
Charles Rodrigues Bastos ${ }^{1}$, Fernando Flores Cardoso ${ }^{2}$, Marcos Jun Iti Yokoo ${ }^{2}$ \\ e Sandro da Silva Camargo ${ }^{1}$ \\ ${ }^{1}$ Universidade Federal do Pampa - Avenida Maria Anunciação Gomes de Godoy, $n^{\circ} 1650$, Bagé, RS, Brasil \\ ${ }^{2}$ Empresa Brasileira de Pesquisa Agropecuária - Rodovia BR-153, Km 633, Vila Industrial, Bagé, RS, Brasil
}

\begin{abstract}
RESUMO
Valores extremos podem distorcer o resultado de uma avaliação genética, assim como a exclusão destes valores pode ocultar alterações relevantes em um rebanho. A predição dos valores genéticos, em uma população de indivíduos, deverá ter um nível mais elevado de precisão quando a informação fenotípica e de pedigree disponíveis corresponderem a dados fidedignos. Entretanto, fatores como o efeito potencial de lesões desconhecidas, tratamento diferenciado, doenças, ou até mesmo erros de entrada de dados são variáveis que não são consideradas nos modelos estatísticos, mas são capazes de comprometer a qualidade dos dados a ponto de influenciar significativamente o desempenho de um indivíduo, ou grupo de indivíduos, gerando valores extremos que poderão enviesar as estimativas dos parâmetros genéticos. Os estimadores estatísticos mistos, como o BLUP, são os mais utilizados para a predição de valores genéticos, porém são sensíveis a dados com valores extremos e necessitam editar ou descartar estes dados, para mitigar a distorção dos resultados. Diante disso, o objetivo deste trabalho é demonstrar que a implementação de um estimador robusto, capaz de reduzir a influência de dados com valores extremos, possibilita a predição de valores genéticos sem descartar dados. Para tanto, foi desenvolvido um algoritmo que calcula as equações de modelos mistos, identifica a relação entre valores extremos e o resultado da predição e introduz, quando necessário, uma variável de ponderação capaz de reduzir o desvio de cada observação em relação à média de sua unidade amostral. Foram avaliadas duas características distintas, em bovinos da raça Brangus, e houve redução de 55 e 79 por cento no intervalo entre o maior e o menor valor estimado. Os resultados obtidos demonstraram que foi possível melhorar a precisão das estimações, reduzindo, em alguns casos, a influência de valores extremos em até 90 por cento, de acordo com o desvio padrão calculado, sem descartá-los da avaliação. Desta forma, diante de conjuntos de dados com valores extremos, o estimador robusto apresentou resultados mais precisos, em comparação ao BLUP.
\end{abstract}

\section{PALAVRAS-CHAVE}

Estimação Genética, Valores Extremos, Melhoramento Genético Animal

\section{INTRODUÇÃO}

É incontestável a relevância do agronegócio no cenário econômico brasileiro. É fato, também, que a pecuária contribui significativamente para o crescimento da economia, sendo fundamental para a manutenção do saldo comercial positivo no último triênio (ABIEC, 2019). Além disso, no ano passado, o Brasil quebrou o recorde de exportações de carne bovina, o que reflete a capacidade de acompanhar a demanda por produtos cada vez mais qualificados, capazes de atender os rigorosos níveis de exigência do mercado internacional.

O destaque brasileiro no cenário mundial é decorrência de fatores positivos como: a ampla extensão territorial, as fontes alimentícias apropriadas para a criação de gado, o melhoramento genético para adaptação dos animais ao meio ambiente e o emprego de tecnologias capazes de aumentar os índices de produtividade (Vicensotti, Montebello e Marjotta-Maistro, 2019). A necessidade de satisfazer os padrões de exigência do mercado consumidor e ainda propiciar lucro ao produtor, motivou a busca por tecnologias capazes de proporcionar a adaptação de rebanhos em diferentes ambientes ou potencializar determinadas características, comercialmente importantes, em um rebanho (Rosa, 2018).

Diante deste contexto, o aprimoramento genético é uma das preocupações dos programas de melhoramento animal e os modelos genéticos estatísticos são os instrumentos utilizados para averiguar se haverá, ou não, evolução de uma ou mais características em um conjunto de indivíduos monitorados (Pereira, 
2008). A predição acurada de valores genéticos é fundamental para o sucesso de um programa de melhoramento animal pois proporciona, ao pesquisador, a oportunidade de promover as alterações hereditárias desejadas, ou seja, garante que os melhores indivíduos possam ser escolhidos para o cruzamento e, desta forma, transmitir os aprimoramentos para as próximas gerações.

Neste contexto, os modelos mistos definidos por Henderson (1973) podem ser implementados em diversas metodologias, com destaque para o Best Linear Umbiased Prediction (BLUP) (Henderson, 1977) que é o estimador mais difundido atualmente. Por definição, estes estimadores abordam tanto os efeitos fixos, ou imutáveis, tais como o sexo ou data do parto, quanto os efeitos aleatórios, ou incertos, tais como peso e altura (Gianola, 1990).

A resolução do BLUP é obtida mediante um sistema de equações lineares, definido como Mixed Model Equations (MME) que, na prática, é viabilizado pela utilização de soluções computacionais (Mrode, 2014). Tal resolução, entretanto, é dificultada pelas dimensões do sistema de equações que precisa ser resolvido e pela sensibilidade a valores extremos que, quando computados, tendem a viesar os resultados da análise (Jiang, 2007). No mesmo sentido, Mrode (2014) considera que grandes volumes de dados podem gerar centenas de milhares de equações, tornando a identificação visual dos valores extremos é impraticável nestes casos, fato que justifica a exclusão de valores, muito afastados da média geral, para que não exerçam influência no resultado da análise.

A mitigação das interferências causadas pelos valores extremos, ou outliers, é abordada por Cardoso et al. (2009) através da proposição de uma metodologia robusta, que não é sensível a outliers, caracterizada pela capacidade de analisar variações, dentro de uma unidade amostral, considerando os dados com valores extremos que seriam desprezados nas MME, pelo fato de estarem muito afastados da média. Para moderar as distorções no resultado final, foi aplicada a metodologia, através da implementação de um algoritmo que considera a inclusão de uma matriz de ponderação que será preenchida com valores proporcionais, obtidos de equação específica, cuja finalidade consiste em redefinir a proporção de influência que um indivíduo com valor extremo será capaz de exercer, na avaliação da média do seu grupo contemporâneo.

Desta forma pretende-se demonstrar, ao final deste trabalho, que a predição dos valores genéticos através de um estimador robusto é capaz de proporcionar resultados mais precisos, em relação à predição com o BLUP, em um conjunto de dados com outliers.

\section{MATERIAIS E MÉTODOS}

Para o presente estudo foram consideradas informações, disponibilizadas pela EMBRAPA Pecuária Sul, de 1552 bovinos da raça Brangus, nascidos entre 1990 e 2018, pertencentes ao seu programa de avaliação genética, o PampaPlus. Programa este que tem como finalidade calcular os valores genéticos e índices, para designar as vantagens obtidas em usar determinado reprodutor ou matriz. A coleta dos dados, disponibilizados para esta pesquisa, segue as normas do PampaPlus (Cardoso, Lopa e Teixeira, 2016), que recomenda a participação de um especialista, indicado pela EMBRAPA, para realizar as aferições, medições e avaliações visuais. As características analisadas, com definições pré-estabelecidas de valores para variância genética $(17,41)$ e variância residual $(30,21)$ foram: a área de olho de lombo (AOL) ao sobreano e a espessura de gordura (EG), considerando, para ambas, os grupos contemporâneos (GC) como efeito fixo. As medidas de AOL e EG são aferidas através de exame de ultrassonografia, descrito em Rosa (2018). Os GC são compostos por: (sexo + lote + ano de nascimento), conforme definição de Cardoso, Lopa e Teixeira (2016).

Os valores para AOL estão expressos em centímetros quadrados $\left(\mathrm{cm}^{2}\right)$, que é a unidade de medida padrão desta característica. O mesmo vale para EG, que está expressa em milímetros $(\mathrm{mm})$. Foi considerado o teste de normalidade de Shapiro Wilk, para calcular o valor de $p$, assumindo que um valor de $p$ inferior a 0,05 indica que os dados não possuem uma distribuição normal (Shapiro e Wilk, 1965). A Figura 1 mostra a representação dos valores de AOL e EG através de histogramas individuais, com valores de $p=3.416 \mathrm{e}-10$ e $p=2.2 \mathrm{e}-16$, respectivamente. A definição de outliers obedeceu ao padrão definido em Cardoso, Lopa e Teixeira (2016), que é de 3,5 desvios padrão. Desta forma, foram identificadas 3 observações que extrapolaram os limites para a AOL e 9 observações para EG. 

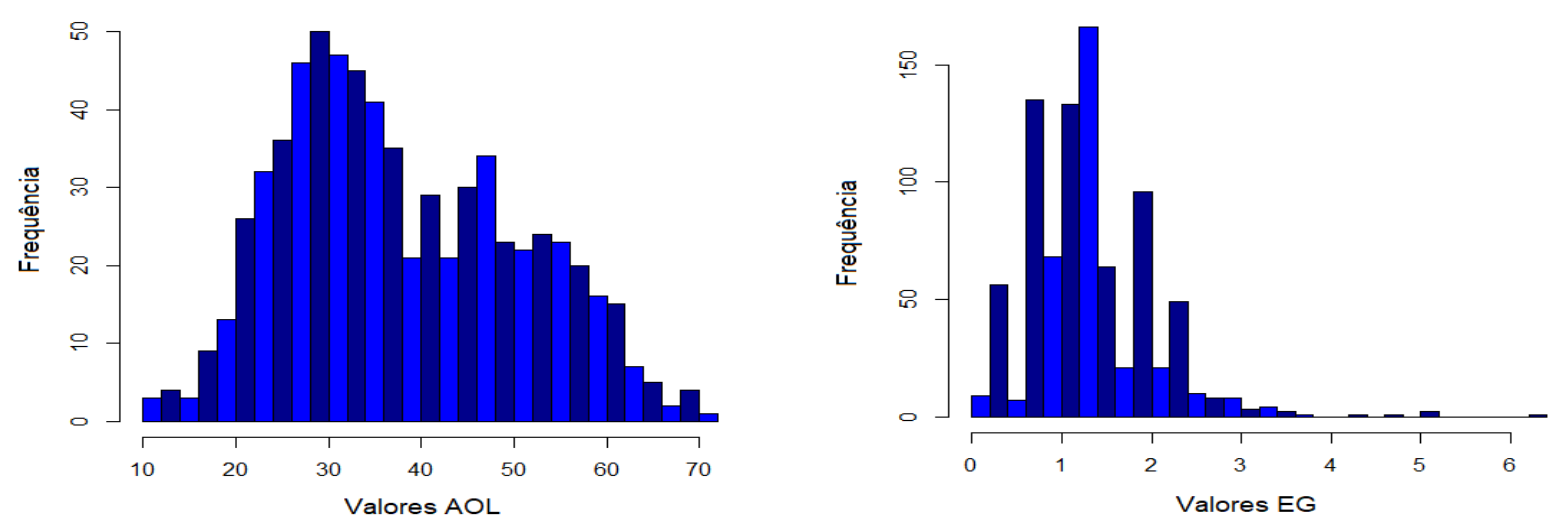

Figura 1. Histograma dos valores fenotípicos para as características AOL e EG

O algoritmo desenvolvido é capaz de receber os dados fenotípicos e de pedigree, estimar os valores genéticos de acordo com o modelo animal e avaliar os resultados quanto à presença de outliers. Além disso, é capaz de calcular as variâncias e acurácias da predição. A caracterização de quais dados são, ou não, outliers, foi ajustada de acordo com as definições da EMBRAPA, mas pode ser redefinida, conforme a necessidade do pesquisador. O fluxo de execução do algoritmo considera as etapas estabelecidas para: leitura e interpretação dos dados, cálculo das equações de modelos mistos, análise e mitigação de valores extremos.

Para adaptar os dados de entrada, predizer os valores genéticos e mitigar as deformidades causadas pelos outliers, foi utilizado o software $\mathrm{R}$ (Team, 2013), na versão 3.5.2, complementado pela biblioteca pedigreemm (Vazquez et al., 2010) versão 0.3-3, que possibilitou o cálculo da matriz de parentesco. Para a geração dos gráficos utilizados neste trabalho, foram utilizadas as bibliotecas plotly (Sievert et al., 2017), na versão 4.9.0 e ggplot2 (Wickham , 2011), na versão 3.2.1. Para a execução dos testes e análises de desempenho foi utilizado um servidor, com o sistema operacional Ubuntu versão 16.04.5, com 8Gb de memória RAM e processador octacore de 64 bits Intel(R) Xeon(R) CPU E7- 4830 @ 2.13GHz.

O procedimento adotado foi a aplicação do BLUP sobre o modelo animal, seguido do cálculo do erro padrão da predição e reajuste das variáveis, alocadas em uma matriz de ponderação, capazes de reduzir ou preservar a significância de cada observação dentro do conjunto. Este processo foi repetido, até que os valores convergissem com o resultado anterior.

A padronização dos dados de entrada obedeceu às convenções de Henderson (1976), que consistem em organizar o conjunto de animais, de forma que todos os ascendentes precedam suas progênies e, codificá-los, em ordem numérica e exclusiva. Além disso, foi observada a existência de parentais conhecidos e aplicadas as regras apropriadas para cada caso. $\mathrm{O}$ vetor de valores fenotípicos, assim como as matrizes de incidência são extraídos do conjunto de dados, após a padronização.

A matriz de parentesco é capaz de estimar a proporção de genes idênticos entre indivíduos provenientes de um mesmo ascendente. Neste caso, quanto maior a quantidade de genes em comum, maior será o grau de parentesco e a semelhança de desempenho. A montagem da matriz de parentesco, detalhada em Mrode (2014), tem seu ponto de partida na distribuição de valores, entre 0 e 1 , referentes ao coeficiente de endogamia, onde 0 significa nenhuma correlação e 1 significa $100 \%$ de correlação entre os alelos. $\mathrm{Na}$ diagonal principal $\left(a_{i j}\right)$, onde $i=j$, como são comparados os valores do indivíduo com ele mesmo, sempre será atribuído $100 \%$ de endogamia, ou seja, 1. Em seguida, o valor atribuído para cada indivíduo ( $i$ ) é igual a $(1+F i)$, onde $F i$ é o coeficiente de endogamia do indivíduo $(i)$. A matriz gerada é quadrada e os demais elementos, fora desta diagonal, são referentes ao coeficiente de parentesco entre os indivíduos. O BLUP utiliza a inversa da matriz obtida pelos cálculos dos elementos acima. Para tanto, foi considerado o método para preenchimento da matriz quadrada $(n \times n) A^{-1}$, publicado por Henderson (1976), que elimina a necessidade de calcular a inversão.

A resolução da MME consiste em uma série de etapas, capazes de minimizar a variância do erro de predição, estabelecer os efeitos fixos de um determinado atributo e os valores genéticos para cada indivíduo (Jiang, 2007). A notação matricial que descreve o modelo animal é representada pela Equação 1:

$$
\gamma=X \beta+Z \alpha+\epsilon
$$


Onde:

- $\quad \gamma \cdot(n \times 1)$ vetor de observações;

- $\quad \beta:(p \times 1)$ vetor dos efeitos fixos;

- $\quad \alpha:(q \times 1)$ vetor dos efeitos aleatórios;

- $\epsilon:(n \times 1)$ vetor de erros aleatórios;

- $\quad X$ : matriz de incidência $(n \times p)$ que relaciona registros a efeitos fixos;

- $\quad Z$ : matriz de incidência $(n \times q)$ que relaciona registros a efeitos aleatórios;

- $\quad n$ : número de registros;

- $\quad p$ : número de níveis para efeitos fixos;

- $q$ : número de níveis para efeitos aleatórios.

Desta forma, $\gamma$ é o vetor que corresponde aos valores fenotípicos observados nos animais, ou seja, contém tantas linhas quanto forem o número de animais $(n)$ e seus valores são referentes às medições da característica observada. Neste caso, a área de olho de lombo (AOL) ou a espessura de gordura (EG). $\beta$ é um vetor, de dimensões ( $p \times 1)$, que corresponde aos efeitos fixos, ou efeitos ambientais identificáveis, enquanto $p$ é o número de níveis destes efeitos fixos. Neste caso, como o efeito correspondente é o grupo contemporâneo (GC), $\beta$ terá 32 níveis para AOL e 52 níveis para EG. $\alpha$ corresponde ao vetor de efeitos aleatórios, ou genéticos dos animais, tem dimensão $q \times 1$, onde $q$ é o número de níveis para estes efeitos, ou seja, a quantidade de animais com fenótipos no conjunto. $\epsilon$ é um vetor, de dimensões $n \times 1$, correspondente aos efeitos não contemplados no modelo e efeitos aleatórios. Neste caso, $n$ corresponde à quantidade de indivíduos no conjunto, ou seja, todos os animais do conjunto. Finalmente, $X$ e $Z$ são matrizes de incidência, ou delineamento, e só contém valores 0 e 1 . $X$ tem dimensão $n$ x $p$ e relaciona os registros a efeitos fixos, enquanto $Z$ tem dimensão $n \times q$ e relaciona seus registros aos valores genéticos dos animais. As MME sob o modelo animal, conforme Mrode (2014), podem ser formadas de acordo com o sistema de equações 2 :

$$
\left(\frac{\beta}{\alpha}\right)=\left(\frac{\left(X^{\prime} X\right)\left(X^{\prime} Z\right)}{\left(Z^{\prime} X\right)\left(Z^{\prime} Z+A^{-1} \alpha\right)}\right)^{-1}\left(\frac{X^{\prime} \gamma}{Z^{\prime} \gamma}\right)
$$

Onde: $A^{-1}$ representa a inversa da matriz de parentesco e $\alpha$ é o quociente de variâncias ambiental e genética, definido por $\alpha=\sigma^{2}{ }_{\mathrm{e}} / \sigma^{2}$ a, respectivamente. A resolução do modelo misto por inversão da matriz de coeficientes gera um vetor, resultante do desdobramento da equação, de onde podem ser extraídos, nas primeiras linhas, os valores estimados para o efeito fixo (GC) e, logo a seguir, os valores estimados dos efeitos genéticos para cada indivíduo. A matriz de ponderação é construída através da distribuição, em sua diagonal principal, de valores referentes ao ajuste do grau de relevância dos resultados estimados. Possui dimensões $(n \times n)$, onde $n$ é o número de indivíduos com fenótipos do conjunto, e todo o restante dos seus campos possui valor zerado. Segundo Cardoso et al. (2009), sua inclusão no sistema de equações do modelo misto possibilita minimizar o impacto, causado no resultado da análise, por observações com valores extremos. O preenchimento da matriz de ponderação é um processo repetitivo, dado pelo cálculo do erro padrão da estimação, que deve ser refeito a cada iteração. Entretanto, a regra é sempre a mesma: preenchimento da diagonal principal com o valor da variável de ponderação e o restante dos campos zerados. A obtenção do valor, referente à variável ponderação $(d)$ é baseada na Equação 3:

$$
d=\mu /\left(\mu+\epsilon^{2} /(\sigma * \nu)\right)
$$

onde:

- $\quad \mu$ : significa o número de observações consideradas no modelo;

- $\sigma$ : corresponde ao coeficiente de herdabilidade;

- $\quad v$ : é relativo aos graus de liberdade da distribuição $t$;

- $\quad \epsilon$. representa o valor do erro padrão da predição, e é obtido através da Equação 4:

$$
\epsilon=\gamma-X * R[1: p]-Z * R[p+1: n]
$$


onde:

- $\quad \gamma$. é o vetor de valores fenotípicos;

- $X$ : é a matriz de incidência referente aos efeitos fixos;

- $R$ : é o vetor de resultados;

- $\quad R[1: p]$ : é um vetor com os valores genéticos dos grupos, ou efeitos fixos;

- $\quad p$ : é a quantidade de níveis para efeitos fixos;

- $\quad Z$ : é a matriz de incidência para efeitos aleatórios;

- $R[p+1: n]$ : é um vetor, referente aos valores genéticos individuais;

- $n$ : é a quantidade de indivíduos.

Neste sentido, adaptando a equação anterior com a inclusão da matriz de ponderação $(D)$, teríamos a seguinte sentença:

$$
\left(\frac{\beta}{\alpha}\right)=\left(\frac{\left(X^{\prime} D X\right)\left(X^{\prime} D Z\right)}{\left(Z^{\prime} D X\right)\left(Z^{\prime} D Z+A^{-1} \alpha\right)}\right)^{-1}\left(\frac{X^{\prime} \gamma}{Z^{\prime} \gamma}\right)
$$

Desta forma, por meio da Equação 5, os resultados mais acurados terão os seus valores multiplicados por uma variável de ponderação próxima de 1, enquanto valores menos acurados deverão ser multiplicados por uma variável de ponderação mais próxima de zero. Após algumas iterações, as variáveis de ponderação deixam de promover alterações significativas e os resultados tendem a convergir determinando, assim, o encerramento do laço de repetição.

\section{RESULTADOS E DISCUSSÃO}

O cálculo da MME, para predição dos valores genéticos, apresentou resultados que determinaram a existência de outliers no conjunto. A Figura 2 é composta por 3 gráficos que representam a distribuição dos valores genéticos estimados para AOL, de acordo com a quantidade de iterações, onde cada ponto corresponde a um indivíduo e cada círculo delimita um desvio padrão.

O primeiro gráfico corresponde à distribuição dos valores estimados após o cálculo da MME. Neste gráfico, a maioria dos elementos ficou concentrada entre o círculo principal, cujo valor é zero, e os primeiros círculos concêntricos subsequentes, que representam a diferença de um desvio padrão, para mais ou menos. A quantidade de pontos entre os círculos diminui, na medida em que se afastam do círculo principal. A diminuição é gradativa, até restarem apenas três pontos distribuídos em regiões que extrapolam os limites de 3,5 desvios padrão. Dada a existência de outliers, uma nova execução do algoritmo refaz o cálculo da MME considerando, para tanto, a inclusão da matriz D na equação. Este procedimento atribui um grau de relevância, compreendido entre 0 e 1, para cada um dos elementos. Desta forma, um valor muito próximo do círculo principal teve um multiplicador próximo de 1, preservando o seu potencial de relevância. Entretanto, valores mais afastados do círculo principal tiveram os seus multiplicadores proporcionalmente mais próximos de 0. Como consequência, os valores genéticos de todo o conjunto tendem a aproximar-se do círculo principal do gráfico.

O segundo gráfico mostra, sobrepostos aos valores estimados na primeira execução, a distribuição dos valores genéticos estimados após nova execução do algoritmo e recálculo da MME com a inclusão da matriz de ponderação. Apenas uma iteração foi suficiente para que não restassem valores extremos na distribuição dos dados.

O terceiro gráfico mostra uma comparação entre os resultados da predição, os resultados após a primeira iteração, quando foram eliminados os outliers e os resultados finais, após a última iteração, quando houve a convergência dos valores. 


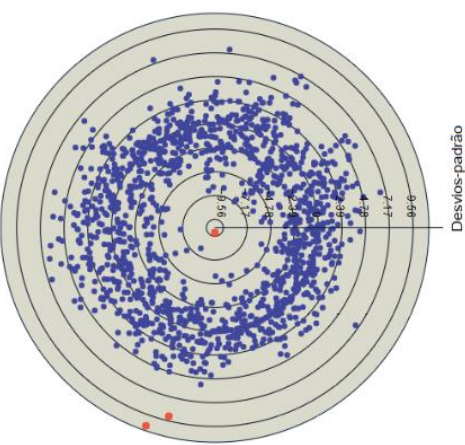

- Distribuição de vlores até 3,5 desvios padrão - Valores extremos
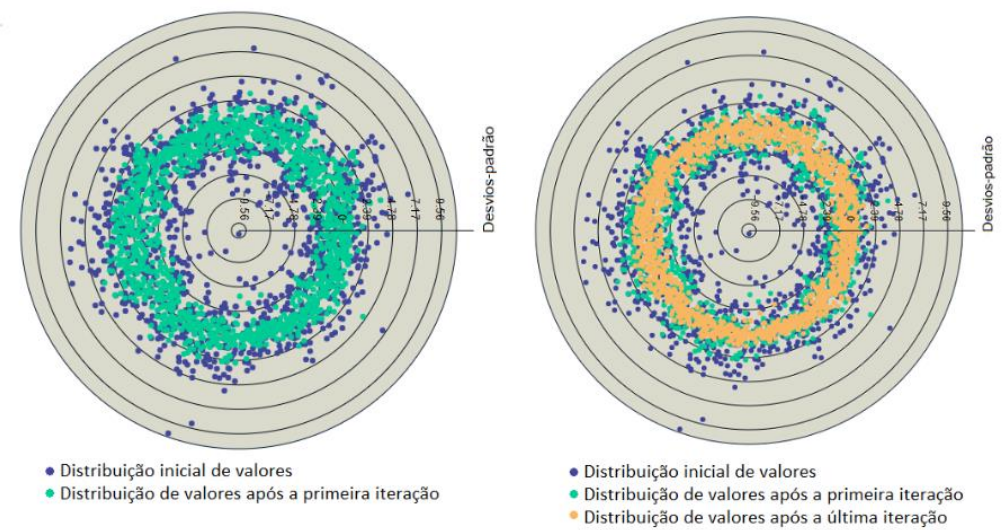

Figura 2. Mitigação dos valores extremos conforme o avanço das iterações

Apesar de uma única iteração ter sido suficiente para que os valores individuais do conjunto deixassem de ser considerados extremos, é factível ao pesquisador definir a quantidade de desvios padrão aceitáveis, de acordo com o nível de confiança e necessidades da pesquisa. Da mesma forma, é possível definir que o algoritmo suspenda a execução ou prossiga, de maneira iterativa, após o ajuste dos outliers.

Neste sentido, para demonstrar esta funcionalidade, foi definido que a etapa de cálculo do erro e recálculo da MME com a matriz $D$ fosse repetida enquanto houvesse diferença significativa entre os efeitos estimados dos grupos contemporâneos em iterações consecutivas. O critério para definir a convergência é o erro absoluto. Entretanto, é factível ao pesquisador definir um limite de casas decimais para que os resultados sejam considerados convergentes.

A introdução da matriz de ponderação, no cálculo da MME, foi capaz de mitigar as distorções geradas pelos valores extremos. Por consequência, a iteração do algoritmo por meio do recálculo do erro residual e das variáveis de ponderação, proporcionou novos ajustes, no que diz respeito a concentração dos resultados mais próximos ao círculo principal, até que fosse estabelecido o fim da execução ou os resultados fossem considerados convergentes. Para ilustrar o processo de mitigação dos valores extremos foi destacado um indivíduo, de forma isolada, cujos valores e seus respectivos ajustes são mostrados na Figura 3.

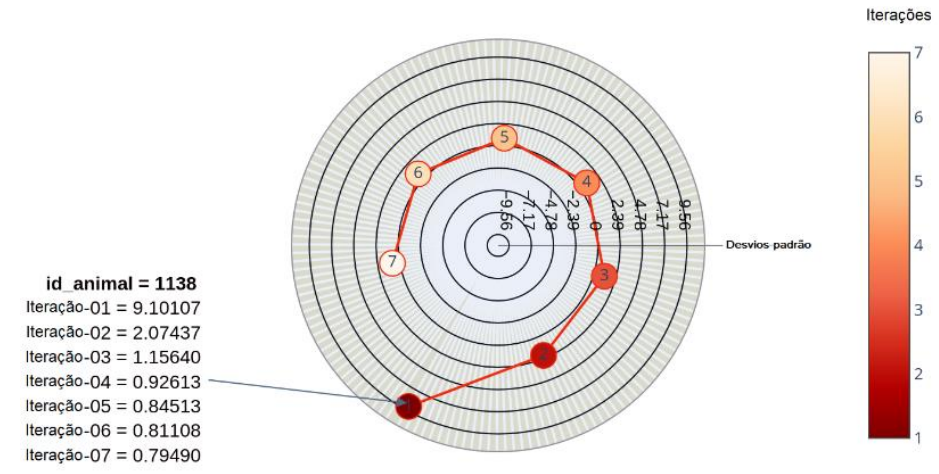

Figura 3. Estimação dos valores genéticos do animal id = 1183 ao longo das iterações

$\mathrm{O}$ animal com id $=1138$ foi considerado um outlier e o seu valor genético original, calculado pela MME, sofreu uma redução no fator de impacto. Ou seja, ao invés de computar $100 \%$ do valor genético calculado, para a composição da média geral do seu grupo contemporâneo, o indivíduo em questão passou a contribuir com um percentual reduzido. Este percentual é proporcional ao desvio padrão encontrado e continua sendo reduzido a cada iteração. De maneira semelhante, os grupos contemporâneos também têm seus efeitos ajustados a cada iteração, devido à modificação dos valores genéticos dos indivíduos que compõem o grupo. A Figura 4 mostra a convergência das médias de valores genéticos para os grupos contemporâneos (030609 29 e 32) ao longo de cada iteração. 


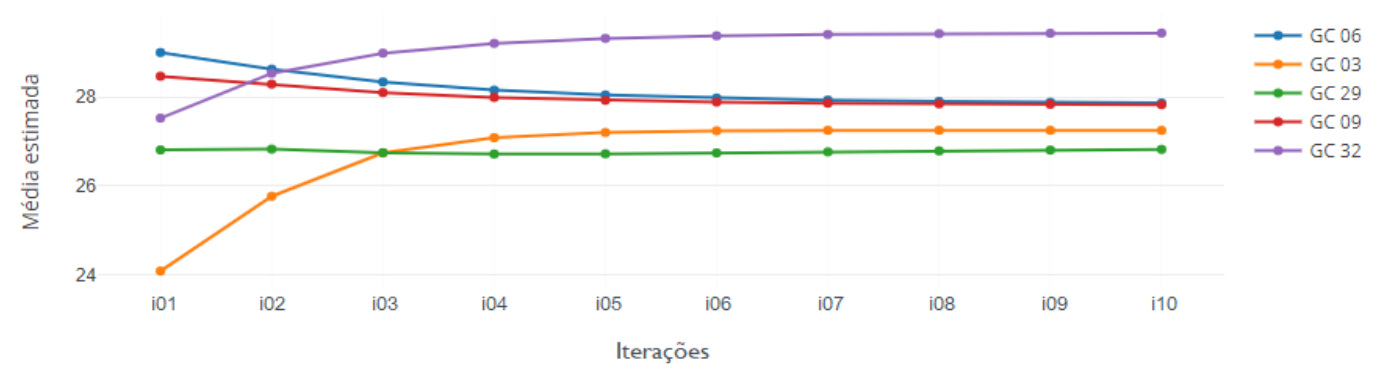

Figura 4. Estimação das médias dos valores genéticos separadas por grupos contemporâneos

O ajuste dos outliers impactou na diminuição do desvio padrão para as características avaliadas. Para ilustrar este impacto, a Figura 5 mostra a redução gradativa na amplitude de distribuição dos indivíduos, de acordo com o valor genético estimado, através de 3 histogramas representando os valores estimados para EG, dispostos conforme a quantidade de iterações. No primeiro histograma a curva de normalidade, representada pela linha vermelha, possui caudas mais longas e vai de $-0,47$ até 1,29 . No segundo histograma, que representa a primeira iteração, os valores vão de $-0,42$ até 0,70 . No último histograma, quando os valores convergiram, a linha representa uma distribuição com a cauda mais curta, variando entre -0,42 e 0,55.
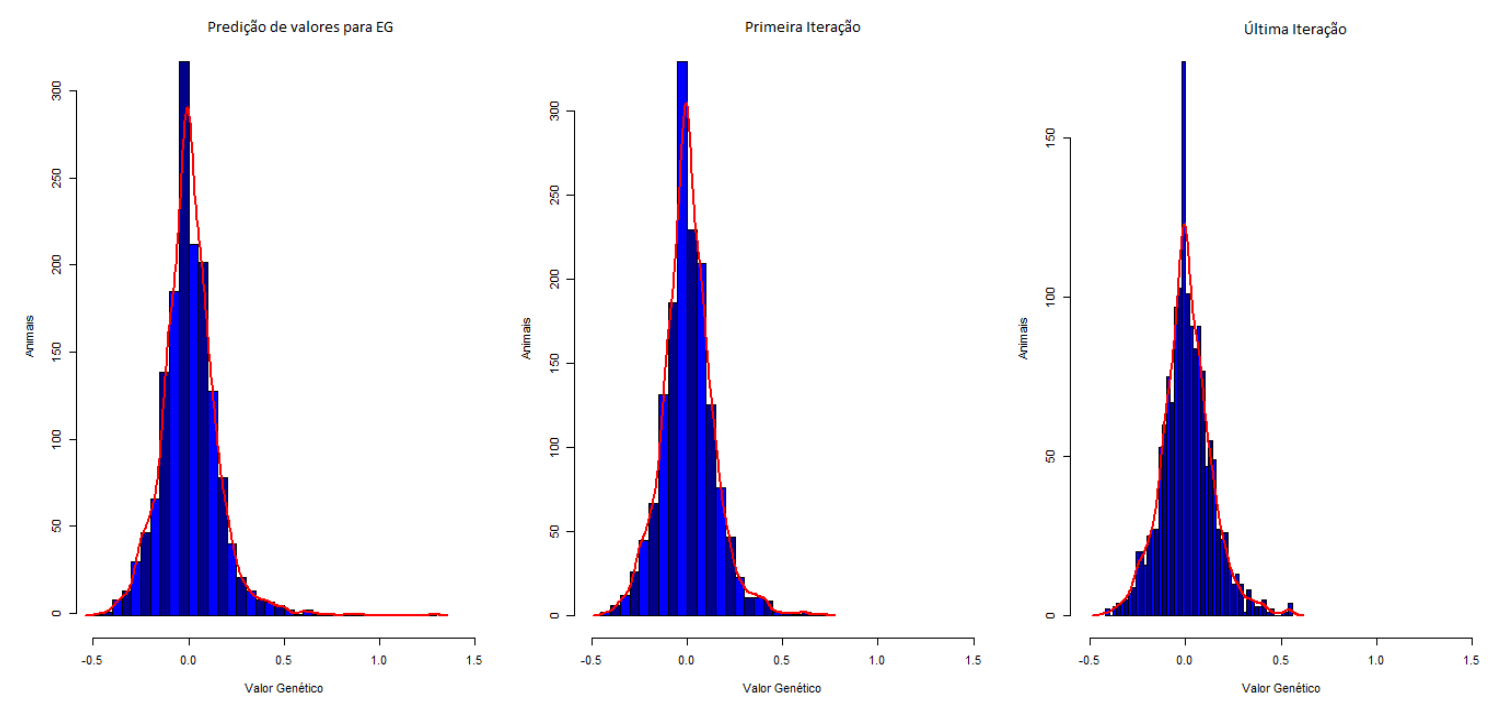

Figura 5. Histogramas dos valores individuais preditos para EG

\section{CONCLUSÕES}

O estudo mostra que, utilizando o estimador robusto, pode-se proceder a avaliação genética sem o descarte de dados considerados outliers. O processo iterativo, caracterizado pelo recálculo do erro padrão e variáveis de ponderação após a apuração dos resultados, também foi capaz de ajustar os efeitos dos GC. A cada iteração os valores foram alterados, mas a proporção desta alteração foi cada vez menos significativa do ponto de vista do deslocamento dos resultados até a média, até que viessem a convergir.

Diante disso, baseado no fato de que a distorção dos resultados de avaliação, ocasionada pelos outliers, pode prejudicar o progresso genético das populações de bovinos de corte, sugerindo valores estimados sem a devida precisão, é de fundamental importância que se considere esta interação nas avaliações genéticas que 
envolvem MME. Principalmente quando se trata da análise de um conjunto massivo de dados, onde a diferença nos resultados pode ser pouco perceptível, dado o volume de informações e a proporção destas distorções, fazendo com que o material genético ou mesmo animais geneticamente inferiores tenham prioridade na seleção.

\section{REFERÊNCIAS}

ABIEC (2019), Perfil da pecuária no Brasil, Associação Brasileira das Indústrias Exportadoras de Carnes, São Paulo, Brasil.

Cardoso, F. F., Lopa, T. M. B. P., \& Teixeira, B. B. (2016). PampaPlus: Avaliação Genética Hereford e Braford. Embrapa Pecuária Sul, Bagé, Brasil.

Cardoso, F. F., Rosa, G. J. D. M., Tempelman, R. J., \& Torres Junior, R. A. D. A. (2009). Modelos hierárquicos bayesianos para estimação robusta e análise de dados censurados em melhoramento animal. Revista Brasileira de Zootecnia, 38(SPE), p 72-80.

Gianola, D., Im, S., \& Macedo, F. W. (1990). A framework for prediction of breeding value. In: Advances in statistical methods for genetic improvement of livestock, p 210-238. Springer, Berlin, Heidelberg.

Henderson, C. R. (1973), Sire evaluation and genetic trends, Journal of Animal Science, Symposium 1973, p10-41.

Henderson, C. R. (1976). A simple method for computing the inverse of a numerator relationship matrix used in prediction of breeding values. Biometrics, Vol. 32, No. 1, p 69-83.

Henderson, C. R. (1977), Best linear unbiased prediction of breeding values not in the model for records, Journal of Dairy Science 60 (5), p 783-787.

Jiang, J. (2007). Linear and generalized linear mixed models and their applications, Springer Science \& Business Media, California, EUA.

Mrode, R. A. (2014), Linear models for the prediction of animal breeding values. Cabi, Boston, EUA.

Pereira, J. C. C. (2008), Melhoramento genético aplicado à produção animal, Editora FEPMVZ, Belo Horizonte, Brasil.

Rosa, A. d. N. (2018), Melhoramento genético aplicado em gado de corte, Empresa brasileira de pesquisa agropecuária, Brasília, Brasil.

Shapiro, S. S., \& Wilk, M. B. (1965). An analysis of variance test for normality (complete samples). Biometrika, 52(3/4), p 591-611.

Sievert, C., Parmer, C., Hocking, T., Chamberlain, S., Ram, K., Corvellec, M., \& Despouy, P. (2017). plotly: Create Interactive Web Graphics via 'plotly. js'. R package version, 4(1), $\mathrm{p} 110$.

Team, R. C. (2013). R: A language and environment for statistical computing.

Vazquez, A. I., Bates, D. M., Rosa, G. J. M., Gianola, D., \& Weigel, K. A. (2010). An R package for fitting generalized linear mixed models in animal breeding. Journal of animal science, 88(2), p 497-504.

Vicensotti, J. M., Montebello, A. E. S., \& Marjotta-Maistro, M. C. (2019), Competitividade brasileira no comércio exterior da carne bovina, Revista IPecege 5(1), 7-18.

Wickham, H. (2011). Ggplot2. Wiley Interdisciplinary Reviews: Computational Statistics, 3(2), p 180-185. 